\title{
Most Recent
}

National Cancer Institute

\section{Source}

National Cancer Institute. Most Recent. NCI Thesaurus. Code C25577.

Nearest to the present. 